\title{
Etat de fertilité des sols du périmètre irrigué gravitaire de Gouran dans la vallée du Sourou au Burina Faso : Constats et perspectives
}

\author{
Adjeffa EPOLYSTE ${ }^{1 *}$, Hamma YACOUBA ${ }^{1}$ et Prosper N. ZOMBRE ${ }^{2}$ \\ ${ }^{1}$ Institut International d'Ingénierie de l'Eau et Environnement, BP594 Ouagadougou 01, Burkina Faso. \\ ${ }^{2}$ Laboratoire de Science de Sol et de l'Environnement, Université de Ouagadougou, \\ BP 7021 Ouagadougou 03, Burkina Faso. \\ *Auteur correspondant, E-mail: epolyste@yahoo.fr, Tél :0023566395096
}

\section{RESUME}

Un regard sur l'état de fertilité des sols du périmètre irrigué gravitaire de Gouran dans la vallée de sourou au Burkina Faso sur deux campagnes (saison sèche et hivernage) a été effectué en 2013. Trois méthodes ont été retenues : les analyses de sols, les essais de fertilité et l'impact d'une nappe perchée sur la production agricole. Le niveau de fertilité des sols a été évalué à travers la réponse du riz à cinq (5) traitements (T1 : témoin absolu; T2 : $200 \mathrm{~kg}$ de NPK et $150 \mathrm{~kg}$ d'urée ; T3 : $200 \mathrm{~kg}$ de NPK et $150 \mathrm{~kg}$ d'urée + zinc ; T4 : $200 \mathrm{~kg}$ de NPK et $150 \mathrm{~kg}$ d'urée +5 t/ha de matière organique) et T5:200 kg de NPK et $150 \mathrm{~kg}$ d'urée + enfouissement des souches) afin de déceler les carences possibles en éléments minéraux dans les sols mais aussi d'évaluer la persistance d'une nappe qui engendrerait un engorgement permanent des sols avec son corollaire sur le rendement des cultures. Les analyses des sols attestent une carence en phosphore $(\mathrm{P}<30 \mathrm{ppm})$ et en azote $(\mathrm{N}<5 \%)$. La valeur élevée du rapport $\mathrm{C} / \mathrm{N}$ en moyenne (13), laisse présager une faible minéralisation de la matière organique dans ces sols et nécessite une réaction corrective pour augmenter l'efficience en éléments minéraux. En revanche, on peut dire que l'impact de la nappe sur le rendement des cultures dans le périmètre irrigué gravitaire de Gouran ne présente pas des effets préjudiciables.

(C) 2014 International Formulae Group. All rights reserved.

Mots clés : Périmètre irrigué gravitaire, fertilité des sols, efficience, nappe, Burkina Faso.

\section{INTRODUCTION}

Le Burkina Faso, comme d'autres pays sahéliens, connaît des problèmes d'insécurité alimentaire à cause de la faible productivité agricole. Cela est dû d'une part à la variabilité climatique et surtout à l'appauvrissement des sols dont les causes sont à la fois naturelles et anthropiques. L'état Burkinabé fait de l'irrigation des terres agricoles l'approche permettant de combattre l'insécurité alimentaire. La création du périmètre irrigué de Gouran (540 ha), situé à environ 220 km au nord-ouest de Ouagadougou, poursuit cet objectif. Malheureusement, ce périmètre est aujourd'hui peu performant. De 7 t/ha environ de paddy par campagne pendant les cinq premières années après l'aménagement, les rendements sont passés à 4 t/ha dans les 
dernières années (AMVS, 2010). Les agriculteurs en parlant de la fertilité de sol, affirment que celle-ci baisse dans le périmètre de Gouran. En effet, les pratiques «d'impasses » et du moindre recours aux analyses de terre peuvent entraîner une réduction à moyen terme de la fertilité des sols (Pèlerin et SYS.C, 2009). Les terres connaissent dans le processus de mise en valeur et d'exploitation, une perte d'éléments nutritifs dont l'importance s'accroît au fur et à mesure que le processus dure, avec pour conséquence la chute de rendement. En général, les compensations faites sont inférieures à la perte des éléments nutritifs engendrée par ce processus (Vander Pol, 1994; Boko et Kpagbin, 1996). Au regard de ce constat, la présente contribution a pour objectif d'approfondir les investigations nécessaires pour proposer des solutions techniques durables pouvant améliorer la fertilité des sols. De façon spécifique, il s'agit d'évaluer le niveau de fertilité actuel des sols du périmètre de Gouran à travers un sondage pédologique, une analyse des indicateurs physico-chimiques et une instrumentation piézométrique du périmètre pour cerner l'effet de la nappe sur le rendement et de mettre en place des essais de fertilité afin de déterminer le meilleur traitement pour optimiser les rendements.

\section{MATERIEL ET METHODES}

\section{Localisation du site d'étude}

Le périmètre irrigué de Gouran (540 ha) est situé à proximité du village Gouran à environ $220 \mathrm{~km}$ au nord-ouest de Ouagadougou, $41 \mathrm{~km}$ à l'ouest de Tougan et à $7 \mathrm{~km}$ au sud-ouest de Niassan. Administrativement, l'aménagement hydro agricole de Gouran est situé dans le département de Lanfiera, Province de Sourou dans la région de la boucle de Mouhoun (Figure 1). L'accès au périmètre est assuré par la route latéritique R17 reliant la localité de
Tougan à celle de Lanfiera sur $41 \mathrm{~km}$ puis la route latéritique reliant Lanfiera à Di jusqu'au village de Gouran sur environ $4 \mathrm{~km}$. A partir du village de Gouran, le périmètre est accessible par une piste en terre. Les Figures 1et 2 montrent respectivement la localisation de la région et du périmètre.

\section{Méthode}

La méthode adoptée pour mener cette étude a consisté à croiser quatre informations qui pourraient concourir toutes à mieux diagnostiquer l'état de la fertilité dans le périmètre et proposer des solutions d'amélioration. Il s'agit i) d'un essai de fertilité, ii) de sondage pédologique, iii) d'analyse de sols et iv) de suivi de la nappe phréatique. Les méthodes adoptées pour l'acquisition de chacune de ces quatre informations sont décrites dans les paragraphes ci-dessous.

\section{Essai de fertilité}

Il participe au diagnostic de la fertilité des sols et permet d'évaluer le stock de nutriments de ces sols, de déterminer les éléments limitant le rendement des cultures et d'estimer les besoins de ces cultures. A l'issue de ce test, des informations collectées sont exploitées pour formuler des recommandations en vue d'augmenter le rendement.

La conduite des essais sur le terrain a été effectuée suivant le dispositif composé de 4 blocs avec 5 traitements. Chaque bloc est composé de 5 parcelles élémentaires. Une parcelle élémentaire a une surface de $20 \mathrm{~m}^{2}$ (5 $\mathrm{m} \times 4 \mathrm{~m})$.

L'expérimentation est réalisée avec la variété de riz NERICA FKR $62 \mathrm{~N}$.

Les paramètres mesurés sont les rendements en paddy et en paille.

\section{Objets}

T1: témoin absolu : 
T2: $200 \mathrm{~kg}$ de NPK et $150 \mathrm{~kg}$ d'urée ;

T3 : $200 \mathrm{~kg}$ de NPK et $150 \mathrm{~kg}$ d'urée + Zinc ;

T4 : $200 \mathrm{~kg}$ de NPK et $150 \mathrm{~kg}$ d'urée + 5

$\mathrm{T} /$ ha de matière organique

T5 : $200 \mathrm{~kg}$ de NPK et $150 \mathrm{~kg}$ d'urée + enfouissement des souches

NB : $200 \mathrm{~kg}$ de NPK et $150 \mathrm{~kg}$ d'urée est la dose minérale recommandée et $5 \mathrm{t} /$ ha est la dose de matière organique recommandée.

\section{Sondage pédologique et analyse de sols}

Pour réaliser le sondage pédologique du périmètre irrigué de Gouran, la méthode de transect a été utilisée. Ainsi, un transect s'étendant du sud-est au nord et touchant tous les types de sols du périmètre, a été tracé.

Des profils pédologiques ont été ouverts tout le long du transect et décrits suivant les directives de la FAO (1994). Les couleurs ont été appréciées à l'aide du Code Munsell. Après la description, des échantillons des sols ont été prélevés horizon par horizon des différentes fosses pédologiques ouvertes pour l'analyse physicochimique au laboratoire. Les différentes données sur le profil et son environnement (profondeur, couleur, structure, texture, charge graveleuse, activités biologiques, végétation et/ou système de culture, drainage, etc...) ont été consignées dans des fiches de description élaborées par le BUNASOLS (1989). Après les analyses au laboratoire, les sols ont été identifiés et classés suivant le système de la Commission de Classification Pédologique et de Cartographie des Sols (CPCS, 1967). Quant à la perméabilité, elle est obtenue à partir de la vitesse d'infiltration de l'eau dans les sols mesurée suivant la méthode de Muntz.

\section{Analyse des sols et méthodes}

Après séchage des échantillons à l'air ambiant du laboratoire, de leur broyage et de leur tamisage à $2 \mathrm{~mm}$, les méthodes résumées dans le Tableau 1 ont été utilisées (BUNASOLS, 1987).

Les différents modes opératoires sont sous forme de recueil au BUNASOLS dont les normes de 1990 ont aussi été utilisées pour interpréter les résultats analytiques.

La réserve en eau utile (RU) est déterminée par la formule ci-dessous.

RU = (H pF2,5 - HpF4,2) xZxda / 100

Où :

* RU = Réserve en eau utile $(\mathrm{mm})$;

* da $=$ densité apparente $(=1,7$ en moyenne pour les sols du Burkina Faso);

* $\mathrm{Z}=$ épaisseur de l'horizon sur les 50premiers centimètres;

* HpF 2,5 : humidité du sol à la capacité au champ en $\%$;

* HpF 4,2 : humidité du sol point de

flétrissement permanent en $\%$.

\section{Évaluation de la fertilité des sols du périmètre}

La classe de fertilité est le facteur diagnostic utilisé. Elle est déterminée à partir de:

- La richesse en éléments nutritifs principaux (azote, phosphore, potassium), en matière organique et en bases échangeables ;

- Les indices de disponibilité en éléments nutritifs ( $\mathrm{pH}$ eau et $\mathrm{pHkcl}$ );

- La capacité d'échange cationique et le taux de saturation en bases.

La valeur de chacun de ces paramètres dans le sol est affectée d'une cotation qui va de 1 à 5 . La somme des cotations définit la classe de fertilité (Tableau 2).

Instrumentation piézométrique de la nappe Six (6) stations piézométriques $\left(\mathrm{P}_{1}, \mathrm{P}_{2}\right.$, $\left.\mathrm{P}_{3}, \mathrm{P}_{4}, \mathrm{P}_{5}, \mathrm{P}_{6}\right)$ ont été installées tout le long du transect pour mettre en évidence la présence d'une nappe perchée qui par sa persistance influencerait le rendement des cultures du périmètre. Les piézomètres sont des tubes en pvc de $4 \mathrm{~cm}$ de diamètre implantés suivant 
la profondeur de la nappe par endroit $(70 \mathrm{~cm}$, $78 \mathrm{~cm}, 72 \mathrm{~cm}, 87 \mathrm{~cm}, 30 \mathrm{~cm}$ et $107 \mathrm{~cm})$. Dans leur partie supérieure, ces tubes ont été entourés d'une gaine colmatante de bentonite afin d'éviter les infiltrations d'eau par le haut. La base de ces tubes a été percée sur une hauteur de $30 \mathrm{~cm}$ et entourée de graviers pour empêcher le colmatage. La cote $\mathrm{TN}$ a été levée à chaque point du piézomètre. Un opérateur mesure la profondeur du niveau de l'eau dans le piézomètre chaque semaine et détermine le niveau piézométrique.
L'opération s'est exécutée sur deux campagnes agricoles.

\section{Analyse des données}

Une analyse de variance (ANOVA) a été réalisée à l'aide du logiciel GENTSTAT Discovery version 4. Les moyennes, lorsque des différences étaient mises en évidence, ont été classées à l'aide du test de StudentNewman-Keuls au seuil de signification de $5 \%$.

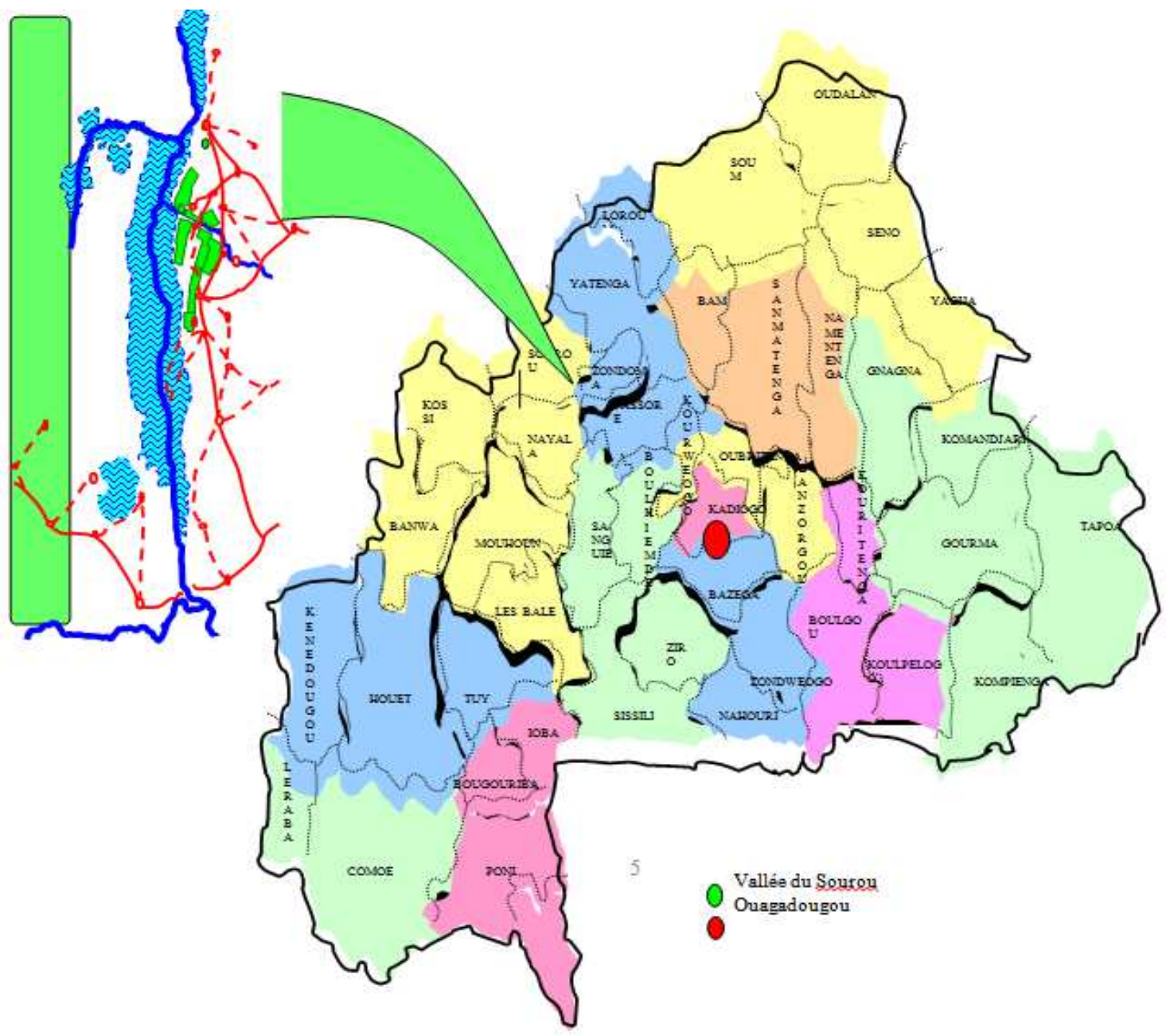

Figure 1 : Localisation de la vallée de Sourou. 


\section{RÉSULTATS ET DISCUSSION}

Caractéristiques morphologiques et hydriques des sols

L'étude a répertoriée six unités taxonomiques au niveau du périmètre de Gouran. Le Tableau 3 indique leurs nomenclatures et leurs proportions. On constate que les BEHV sont majoritaires dans le périmètre de Gouran suivis des BEF. Les restes sont dans des proportions moindres. Cela corrobore les résultats de BUNASOL (1988) et ceux d'ORSTOM, (1976) sur le de périmètre Débé.

Les analyses granulométriques de ces sols, l'évaluation de leur réserve en eau et leur infiltrabilité sont résumées dans le Tableau 4.

On constate que la réserve en eau est élevée dans les sols hydromorphes suivi des vertisols mais elle est faible dans les sols ferrugineux.

Les sols ferrugineux (FLTC, BEF) sont profonds $(>70 \mathrm{~cm})$, bruns en surface avec une texture limono-argileuse et bruns jaunâtre avec une texture argileuse en profondeur. Modérément drainés, leur charge graveleuse apparaît à partir de $65 \mathrm{~cm}$ et est constituée de concrétions ferrugineuses. La structure est massive en surface, polyédrique subangulaire en profondeur. La vitesse moyenne d'infiltration de l'eau est autour de $0,9 \mathrm{~cm} / \mathrm{h}$. L'activité biologique est bien marquée.

Les sols hydromorphes (BEH, HPGS, BEHV) ont une texture fine de haut en bas du profil (argilo-sableuse à argileuse). Ils sont fermes à l'état frais et mal drainés. La teinte dominante est brun grisâtre foncé. Ils sont caractérisés par un engorgement temporaire des horizons de surface et ou de profondeur. L'hydromorphie est y bien prononcée et se manifeste par des phénomènes d'oxydoréductions. La structure est en général polyédrique subangulaire moyenne et grossière. La vitesse moyenne d'infiltration de l'eau varie autour de $0.20 \mathrm{~cm} / \mathrm{h}$ traduisant une perméabilité lente des sols. Le système racinaire et l'activité biologique sont remarquables. Les vertisols ont une structure polyédrique en surface et deviennent prismatiques en profondeur. La texture est sablo-argilo-limoneuse en surface avec des larges fentes de retrait et argilo- sablolimoneuse en profondeur. Ils sont gris brun foncé en surface et brun olive clair à l'état humide en profondeur.

\section{Caractéristiques physico-chimiques des sols Les sols FLTC, BEHV et VV} présentent des taux de matière organique supérieurs à $1 \%$ contre une valeur idéale de $1,5 \%$ avec des textures à dominance argileuse. Dans les sols BEH, HPGS et BEF, les teneurs en matière organique sont faibles, et inférieures à $1 \%$ avec une décomposition bonne à rapide $(11,66<\mathrm{C} / \mathrm{N}<12)$ alors qu'elle est lente sur les autres sites. Les teneurs en azote sont faibles $(0.043 \%)$ dans tous les types de sol (Tableau 4). Les valeurs en phosphore assimilable sont toutes inférieures au seuil de $30 \mathrm{ppm}$ dans tous les sols du périmètre. Ce qui signifie que les sols du périmètre sont carencés en phosphore et en azote. Par contre, ils sont bien pourvus en potassium disponible mais les valeurs les plus élevées sont observées dans les sols hydromorphes et les vertisols qui par ailleurs sont en général moyennement pauvres en fer total et libre.

Une variabilité des teneurs en bases échangeables s'observe dans les sols (Tableau 5). La texture des sols affecterait la teneur en bases échangeables. Les sols les plus argileux (hydromorphes) ont les valeurs les plus élevés en bases échangeables contre les valeurs les plus basses dans les sols ferrugineux. Les teneurs en calcium et magnésium échangeables y sont fortes mais faibles dans les sols hydromorphes, celles du potassium et du sodium varient de basses à très basses. Les sols ferrugineux sont moyennement acides $(5,95<\mathrm{pH}<6,47)$. Ce résultat s'apparente à celui obtenu par Amadji (1999) sur le site de BOCHICON au BENIN, à l'opposé des sols hydromorphes qui sont neutres à légèrement acides. Quant aux vertisols, ils tendent vers la basicité. Avec $85 \%$, les sols ferrugineux accusent un risque de toxicité ferreuse. 


\section{Evaluation de la fertilité des sols}

Après affectation des cotations à chaque type de sol sur la base des paramètres d'évaluation énoncée précédemment (Tableau, 5), les sols du périmètre sont classés comme l'indique le Tableau 6.

Tous les types de sols appartiennent à la même classe de fertilité c'est-à-dire moyenne. Ce résultat confirme ceux de MCA (2010).

En production végétale, la gestion de la fertilité des sols est capitale. En effet, la fertilité est l'aptitude d'un sol à assurer durablement la production et liée à la richesse du sol en éléments minéraux (Lavigne-Delville, 1996). Elle peut augmenter ou décroître en fonction des pratiques culturales. Ainsi, la dégradation chimique des sols est le résultat de l'appauvrissement de ceux-ci en éléments nutritifs. Cette dégradation en zone soudanosahélienne est plus rapide en ce qui concerne les éléments majeurs. L'azote et le phosphore sont les deux facteurs militants de la production en riziculture en général selon Traoré et Toé (2008). Le périmètre de Gouran connaît une déficience en azote et en phosphore. Cela peut expliquer la baisse du rendement dans le périmètre. La déficience en azote est généralement considérée comme la principale cause des baisses de rendement (Narteh et Sahrawat, 1997; Cassman et al., 1998). Le déficit d'azote entraîne la réduction du nombre de talles, réduisant ainsi le nombre de panicules et de grains (Bandaogo, 2010). L'action de l'azote se traduit par le verdissement rapide et la croissance de la végétation (Dobelmann, 1976).

Le phosphore permet une meilleure croissance racinaire, favorise un tallage plus actif avec des talles fertiles et agit sur le bon développement des grains en élevant leur valeur alimentaire. Il avance son épiaison et agit positivement sur sa productivité (Adam, 2000). L'aménagement rationnel des rizières au point de vue hydraulique, l'utilisation des variétés très productives et l'amélioration des techniques culturales sont autant de facteurs d'un meilleur rendement CIRAD - GRET (2006).

En effet, le riz irrigué répond généralement bien à la fertilisation surtout azotée si la lame d'eau est maintenue dès le début de la culture. Aussi, il faut nécessairement relever le niveau de fertilité des sols pauvres et compenser les éléments minéraux exportés par la récolte du grain et de la paille (Dobelmann, 1976) d'où l'importance de la fumure. Une récolte de 100 $\mathrm{kg}$ de paddy exporte, selon que la paille est restituée ou non, 1,1 à $2 \mathrm{~kg}$ d'azote, 0,6 à $1 \mathrm{~kg}$ de $\mathrm{P}_{2} \mathrm{O}_{5}$ et 0,3 à $1,3 \mathrm{~K}_{2} \mathrm{O}$ (Camille, 1997). La restitution des pailles par enfouissement ou sous forme de fumier participe du maintien de la fertilité des rizières. Au regard des valeurs de la CEC, on peut dire que les sols du périmètre ont une capacité élevée de maintenir les bases provenant de leur minéralisation interne et celle liée aux apports extérieurs par la fumure minérale. Le niveau de la CEC élevé (Tableau 4) dans les sols hydromorphes et les vertisols est en rapport avec la texture à prédominance argileuse de ces sols. Les valeurs des conductivités électriques sont faibles $(0,072$ à $0,12 \mathrm{mS} / \mathrm{cm})$, ce qui ne présage aucun risque de salinisation.

\section{Relevés piézométriques}

Les relevés piézométriques des deux campagnes ont été représentés sur des graphiques ci-dessus (Figure 3). Ces relevés permettent d'apprécier l'allure de la fluctuation de la nappe dans chaque piézomètre

L'examen global de relevés
piézométriques montre que le toit
piézométrique présente des fluctuations suivant une courbe concave. Cette concavité est liée au niveau de l'eau dans le sourou et selon le domaine pédologique. Au piézomètre P1 qui couvre le domaine ferrugineux où apparaissent en subsurface des horizons indurés des taches et concrétions, le niveau de l'eau à l'implantation du piézomètre était $0,70 \mathrm{~m}$, au quotidien la fluctuation du niveau 
de ce piézomètre est régressive jusqu'au début $\mathrm{du}$ mois de juin (Figure 3). Il se maintient jusqu'à la fin du mois avant de remonter graduellement pour atteindre le pic vers le mois de novembre. Cette période correspond $\mathrm{au}$ plus haut niveau du sourou où les gestionnaires du barrage de Lery ouvrent les vannes pour l'évacuation de l'excès de l'eau vers le Mali. On peut déduire que la présence de la nappe à cet endroit du domaine ferrugineux n'est que sporadique. Elle correspondrait à une saturation localisée des macropores des horizons de subsurface. En effet, cette situation pourrait être liée au haut niveau du fleuve sourou pendant cette période de crue. Ce haut niveau, quand il se maintient pendant un certain temps, envahit les rives où sont aménagés les périmètres irrigués. La réponse à cette venue d'eau dépend du type de sol et de sa perméabilité fonctionnelle. Les relevés des piézomètres 2, 3, 4 (Figure 3) présentent une similitude avec celui du piézomètre $\mathrm{P} 1$. Le piézomètre $\mathrm{P} 5$ à cause de sa proximité au lit du sourou voit son niveau beaucoup plus relevé mais toujours est-il que quand le niveau du sourou baisse, le niveau piézométrique baisse et inversement (Figure 3). Dans tous les cas, aucun niveau de piézomètre n'affleure en surface. Toutefois, le relevé du niveau dans les sols proche du lit $\mathrm{du}$ sourou semble expliquer un engorgement de ces sols pendant cette période. L'engorgement en raréfiant l'oxygène dans les sols provoque la baisse de perméabilité des tissus racinaires à l'eau (Kramer, 1969). Or une alimentation minérale normale nécessite une bonne aération des racines (Heller, 1969). Dans le périmètre de Gouran, une comparaison entre l'état d'enracinement et le niveau de la nappe montre que le niveau de la nappe évolue en sens inverse que l'enracinement du riz. L'installation du riz correspond au moment où le niveau de la nappe est relevé. Au fur et à mesure que l'enracinement se développe, le niveau de la nappe baisse. Le risque d'engorgement préjudiciable sur les racines est donc faible voir nul. Par contre, le retrait du niveau de la nappe peut s'accompagner des dépôts des sels nuisibles pour les cultures mais l'analyse des sols ne présage pas des concentrations anormales en sels. On peut en définitive dire que l'impact de la nappe sur le rendement des cultures dans le périmètre irrigué gravitaire de Gouran ne présente pas d'effets préjudiciables.

\section{Effets des traitements sur le rendement du riz}

Les résultats de l'analyse de variance ont montré que les traitements présentent en saison sèche une différence hautement significative (Tableau 7). Les meilleurs rendements en paddy et en paille (7329 et $9229 \mathrm{~kg} / \mathrm{ha}$ ) sont obtenus avec le traitement $\mathrm{T} 5$ suivi de $\mathrm{T} 4$.

Le plus faible rendement en paddy et en paille (4415 et $4988 \mathrm{~kg} / \mathrm{ha}$ ) a été obtenu avec le traitement $\mathrm{T} 1$. La différence est aussi hautement significative en saison humide.

Les moyennes affectées d'une même lettre dans une même colonne ne sont pas significativement différentes au seuil de 5\% par la méthode de Duncan.

L'analyse des résultats des rendements montre des différences significatives entre les différents traitements (Tableau 5). La réponse du riz par rapport à l'apport des doses minérales recommandée est très moyenne, soit $+1000 \mathrm{~kg} / \mathrm{ha}$ par rapport au témoin. L'apport du zinc à la dose recommandée entraîne une réponse encore très positive $\mathrm{du}$ riz. $\mathrm{La}$ différence de rendement est de $1522.2 \mathrm{~kg} / \mathrm{ha}$ entre T3 et T2 et de $2526 \mathrm{~kg} / \mathrm{ha}$ entre T1 et T3. La dose de $5 \mathrm{~T} / \mathrm{ha}$ complémentaire de matière organique à la dose recommandée s'accompagne d'une augmentation de rendement, il en va de même de l'apport supplémentaire de souche enfouie. Ce qui signifie que les sols sont en voie d'une dégradation chimique. Les meilleurs rendements sont obtenus avec les traitements qui appellent à la matière organique. Ce qui confirme que les sols accusent une déficience en azote et en phosphore. En effet, le niveau d'alimentation azotée de la plante dépend de 
l'adéquation entre l'évolution des besoins en azote de la plante au cours du cycle et la disponibilité en azote dans le sol. Il est donc nécessaire de définir les stades clés auxquels les engrais azotés doivent être apportés. Selon Yoshida (1981), la formation de la jeune panicule et celle du nombre d'épillets sont sous le contrôle de la nutrition azotée. Les résultats des essais ont montré que les traitements $\mathrm{T} 4$ et $\mathrm{T} 5$ ont donné les meilleurs rendements paddy et paille. Les plus faibles rendements sont obtenus avec le traitement témoin. En effet, l'évolution des rendements laisse voir une hiérarchisation et une importance des éléments $\mathrm{N}$, P et $\mathrm{K}$. Cette évolution montre que les éléments $\mathrm{N}$ et $\mathrm{P}$ sont de façon équivalente, les deux éléments limitatifs de la production du riz dans le périmètre de Gouran. Ces résultats sont en accord avec ceux de FAO (2002), de l'IFDC
(2008) et de Bandaogo (2010). Selon ces auteurs, l'azote et le phosphore sont les éléments majeurs limitant la production céréalière. Selon Segda (2006), l'azote est l'un des éléments les plus importants qui peut être limitant pour la croissance et le développement du riz dans les conditions aussi variées que celles dans lesquelles se pratiquent la riziculture en général. Les résultats de Pieri (1989) ont montré que le phosphore est l'élément le plus déficitaire dans les sols de savane au Sud du Sahara, mais qu'il demeure l'élément le plus facilement maîtrisable par la fertilisation grâce à sa faible mobilité et son faible niveau d'utilisation par les cultures. Un apport d'engrais et de matière organique a donc un effet positif sur le rendement quel que soit le type de sol.

Tableau 1 : Méthodes d'analyses des sols utilisées.

\begin{tabular}{|c|c|}
\hline Paramètres & Méthodes \\
\hline $\mathrm{pH}$ & pH-mètre avec rapport sol/eau $1 / 2,5$ \\
\hline $\begin{array}{l}\text { Granulométrie } \\
\text { (3fractions) }\end{array}$ & $\begin{array}{c}\text { Pipette Robinson/tamisage après oxydation de la matière } \\
\text { organique avec l'eau oxygénée }\end{array}$ \\
\hline Carbone organique & Méthode Walkley et Black (1934) \\
\hline Azote total & Kjeldahl \\
\hline Phosphore & Bray I \\
\hline \multicolumn{2}{|l|}{ Bases échangeables : } \\
\hline $\begin{array}{l}\text { - } \mathrm{Ca} 2+\text { et } \mathrm{Mg} 2+ \\
\text { - } \mathrm{K}+, \mathrm{Na}\end{array}$ & $\begin{array}{c}\text { Acétate d'ammonium et dosage par spectroscopie } \\
\text { d'absorption atomique; } \\
\text { Acétate d'ammonium et dosage par spectroscopie } \\
\text { d'émission à flamme }\end{array}$ \\
\hline
\end{tabular}

Tableau 2: Normes des classes de fertilité des sols.

\begin{tabular}{lccccc}
\hline Classe de fertilité & Très basse & Basse & Moyenne & Élevée & Très élevée \\
\hline $\begin{array}{l}\text { Somme des } \\
\text { cotations }\end{array}$ & $<15,9$ & $16,0-21,9$ & $22,0-27,9$ & $28,0-33,9$ & $>34,0$ \\
\hline
\end{tabular}


Tableau 3: Représentativité des différents domaines.

\begin{tabular}{llcc}
\hline $\mathbf{N}^{\circ}$ & Type de sol & Superficie (ha) & $\mathbf{\%}$ \\
\hline $\mathrm{P}_{1}$ & Sols ferrugineux tropicaux lessivés à taches et concrétions (FLTC) & 20 & 3,7 \\
$\mathrm{P}_{2}$ & Sols bruns eutrophes tropicaux hydromorphes (BEH) & 11,6 & 2,1 \\
$\mathrm{P}_{3}$ & Sols hydromorphes peu humifères à pseudogley de surface (HPGS) & 18,5 & 3,4 \\
$\mathrm{P}_{4}$ & Sols bruns eutrophes tropicaux hydromorphes vertiques (BEHV) & 255,2 & 47,2 \\
$\mathrm{P}_{5}$ & Les vertisols vertiques (V V) & 39,7 & 7,3 \\
$\mathrm{P}_{6}$ & Sols bruns eutrophes tropicaux ferruginisés (BEF) & 195 & 36,1 \\
& & $\mathbf{5 4 0}$ & $\mathbf{1 0 0}$ \\
\hline
\end{tabular}

Tableau 4: Granulométrie, réserve utile en eau et infiltrabilité des sols du périmètre

\begin{tabular}{|c|c|c|c|c|c|c|c|}
\hline \multirow[b]{2}{*}{ Type de sol } & \multirow{2}{*}{$\begin{array}{l}\text { profondeur } \\
\text { en } \mathbf{c m}\end{array}$} & \multicolumn{3}{|c|}{ Granulométrie \% } & \multirow{2}{*}{$\begin{array}{c}\mathrm{RU} \\
(\mathbf{m m} / \mathbf{5 0} \mathbf{c m})\end{array}$} & \multirow{2}{*}{$\begin{array}{c}\text { Infiltrabilité } \\
(\mathbf{c m} / \mathbf{h})\end{array}$} & \multirow[b]{2}{*}{ Observations } \\
\hline & & Argile & Limon & Sable & & & \\
\hline & $0-20$ & 13,3 & 27,4 & 45,1 & & & \\
\hline \multirow[t]{3}{*}{ FLTC } & $20-54$ & 45,1 & 23,5 & 31,3 & 97,2 & 0,9 & Vitesse rapide \\
\hline & $54-70$ & 43,1 & 19,6 & 37,2 & & & \\
\hline & $0-27$ & 21,5 & 25,4 & 52,9 & & & \\
\hline \multirow[t]{3}{*}{$\mathrm{BEH}$} & $27-60$ & 45,1 & 23,5 & 31,3 & 120,2 & 0,02 & Vitesse très lente \\
\hline & $60-78$ & 39,2 & 25,4 & 35,2 & & & \\
\hline & $0-32$ & 29,3 & 27,4 & 43,1 & & & \\
\hline \multirow[t]{3}{*}{ HPGS } & $32-55$ & 43,1 & 23,5 & 33,3 & 117,3 & 0,22 & Vitesse lente \\
\hline & $55-72$ & 43,1 & 21,5 & 35,2 & & & \\
\hline & $0-20$ & 33,3 & 25,4 & 41,1 & & & \\
\hline \multirow[t]{3}{*}{ BEHV } & $20-43$ & 39,2 & 21,5 & 39,2 & 118,2 & 0,3 & Vitesse lente \\
\hline & $43-72$ & 39,2 & 23,5 & 37,2 & & & \\
\hline & $0-15$ & 35,2 & 27,4 & 37,2 & & & \\
\hline \multirow[t]{3}{*}{ VV } & $15-30$ & 43,1 & 21,5 & 35,2 & 103,4 & 0,18 & Vitesse lente \\
\hline & nappe & & & & & & \\
\hline & $0-20$ & 35,2 & 23,5 & 41,1 & & & \\
\hline \multirow[t]{2}{*}{ BEF } & $20-44$ & 50,9 & 19,6 & 29,4 & 91 & 0,88 & Vitesse rapide \\
\hline & $44-107$ & 45,1 & 15,6 & 39,2 & & & \\
\hline
\end{tabular}


Tableau 5: Caractéristiques physico-chimiques des sols du périmètre de Gouran.

\begin{tabular}{lcccccc}
\hline & FLTC & BEH & HPGS & BEHV & VV & BEF \\
\hline Matière organique \% & 1,074 & 0,663 & 0,58 & 1,004 & 1,04 & 0,903 \\
$\mathrm{C}^{++}$total \% & 0,623 & 0,385 & 0,336 & 0,582 & 0,864 & 0,523 \\
$\mathrm{~N}$ total \% & 0,046 & 0,0326 & 0,028 & 0,046 & 0,0665 & 0,042 \\
$\mathrm{C} / \mathrm{N}$ & 13,66 & 12 & 11,66 & 12,66 & 13 & 12 \\
$\mathrm{P}$ assimilable (ppm) & 5,99 & 7,99 & 2,433 & 8,37 & 4,4 & 6,49 \\
$\mathrm{~K}^{+}$disponible (ppm) & 44,39 & 109,83 & 49,91 & 92,06 & 104,55 & 38,47 \\
$\mathrm{Ca}^{++}$(meq/100g) & 7,77 & 13,4 & 14,4 & 15,86 & 14,59 & 8,81 \\
$\mathrm{Mg}^{++}(\mathrm{meq} / 100 \mathrm{~g})$ & 1,77 & 2,56 & 3,23 & 2,68 & 3,04 & 4,58 \\
$\mathrm{~K}^{++}$(meq/100) & 0,14 & 0,29 & 0,29 & 0,28 & 0,3 & 0,22 \\
$\mathrm{Na}^{+}(\mathrm{meq} / 100 \mathrm{~g})$ & 0,04 & 0,09 & 0,09 & 0,08 & 0,09 & 0,08 \\
$\mathrm{~S}$ (meq/100g) & 9,75 & 16,4 & 18 & 18,84 & 18,03 & 11,15 \\
$\mathrm{CEC}$ (meq/100g) & 16,24 & 21,1 & 22,9 & 23,29 & 23,17 & 16,79 \\
$\mathrm{~S} / \mathrm{CEC}(\%)$ & 60 & 77,7 & 78,3 & 81 & 78 & 66,66 \\
$\mathrm{Pheau}(\mathrm{pv}: 1 / 2,5)$ & 5,95 & 6,62 & 7,02 & 7,37 & 7,41 & 6,47 \\
$\mathrm{Phkcl}(\mathrm{PV}: 1 / 2,5)$ & 5,09 & 5,65 & 6,23 & 6,68 & 6,42 & 5,71 \\
$\mathrm{C}(\mathrm{ms} / \mathrm{cm})$ & 0,078 & 0,1 & 0,11 & 0,14 & 0,24 & 0,12 \\
Fer total \% & 3,15 & 2,46 & 2,99 & 2,46 & 2,85 & 3,02 \\
Fer libre \% & 2,21 & 1,61 & 1,72 & 1,97 & 2,1 & 2,57 \\
Fer libre / Fer total & 0,70 & 0,65 & 2,57 & 0,80 & 0,73 & 0,85 \\
\hline
\end{tabular}

Tableau 6 : Evaluation de la fertilité des sols du périmètre de Gouran.

\begin{tabular}{lcccccc}
\hline Type de sols & FLCT & BEH & HPGS & BEHV & VV & BEF \\
\hline Cotation & 23,5 & 27,75 & 26,5 & 27,75 & 27 & 24,5 \\
\hline Classe de fertilité & moyenne & moyenne & moyenne & moyenne & Moyenne & Moyenne \\
\hline
\end{tabular}


Tableau 7 : Effets des traitements sur les rendements du riz.

\begin{tabular}{lcccc}
\hline Traitements & \multicolumn{2}{c}{ Campagne sèche } & \multicolumn{2}{c}{ Campagne humide } \\
\hline & $\begin{array}{c}\text { Rendement } \\
\text { paddy }\end{array}$ & $\begin{array}{c}\text { Rendement } \\
\text { en paille }\end{array}$ & $\begin{array}{c}\text { Rendement } \\
\text { paddy }\end{array}$ & $\begin{array}{c}\text { Rendement en } \\
\text { paille }\end{array}$ \\
\hline T1 & $4415^{\mathrm{a}}$ & $4988^{\mathrm{a}}$ & $4588^{\mathrm{a}}$ & $5975^{\mathrm{a}}$ \\
$\mathrm{T} 2$ & $5754^{\mathrm{b}}$ & $6754^{\mathrm{b}}$ & $6438^{\mathrm{b}}$ & $6812^{\mathrm{ab}}$ \\
$\mathrm{T} 3$ & $6938^{\mathrm{c}}$ & $7850^{\mathrm{c}}$ & $6688^{\mathrm{b}}$ & $8562^{\mathrm{abc}}$ \\
$\mathrm{T} 4$ & $7225^{\mathrm{c}}$ & $8075^{\mathrm{c}}$ & $7556^{\mathrm{b}}$ & $9388^{\mathrm{bc}}$ \\
$\mathrm{T} 5$ & $7329^{\mathrm{c}}$ & $9229^{\mathrm{d}}$ & $7488^{\mathrm{b}}$ & $10219^{\mathrm{c}}$ \\
Signification & $\mathrm{THS}$ & $\mathrm{THS}$ & $\mathrm{THS}$ & $\mathrm{THS}$ \\
CV (\%) & 15,2 & 16,8 & 25 & 28,9 \\
Lsd & 674,2 & 979,4 & 1662 & 2402,7 \\
probabilité & $<0,001$ & $<0,001$ & $<0,006$ & $<0,005$ \\
\hline
\end{tabular}

THS $=$ Très Hautement Significatif $(\mathrm{P}<0.001), \mathrm{HS}=$ Hautement Significatif $(\mathrm{P}<0.01)$,

$\mathrm{S}=$ Significatif $(\mathrm{P}<0.05), \mathrm{LSD}:$ plus petite différence significative,

$\mathrm{CV}=$ coefficient de variabilité.

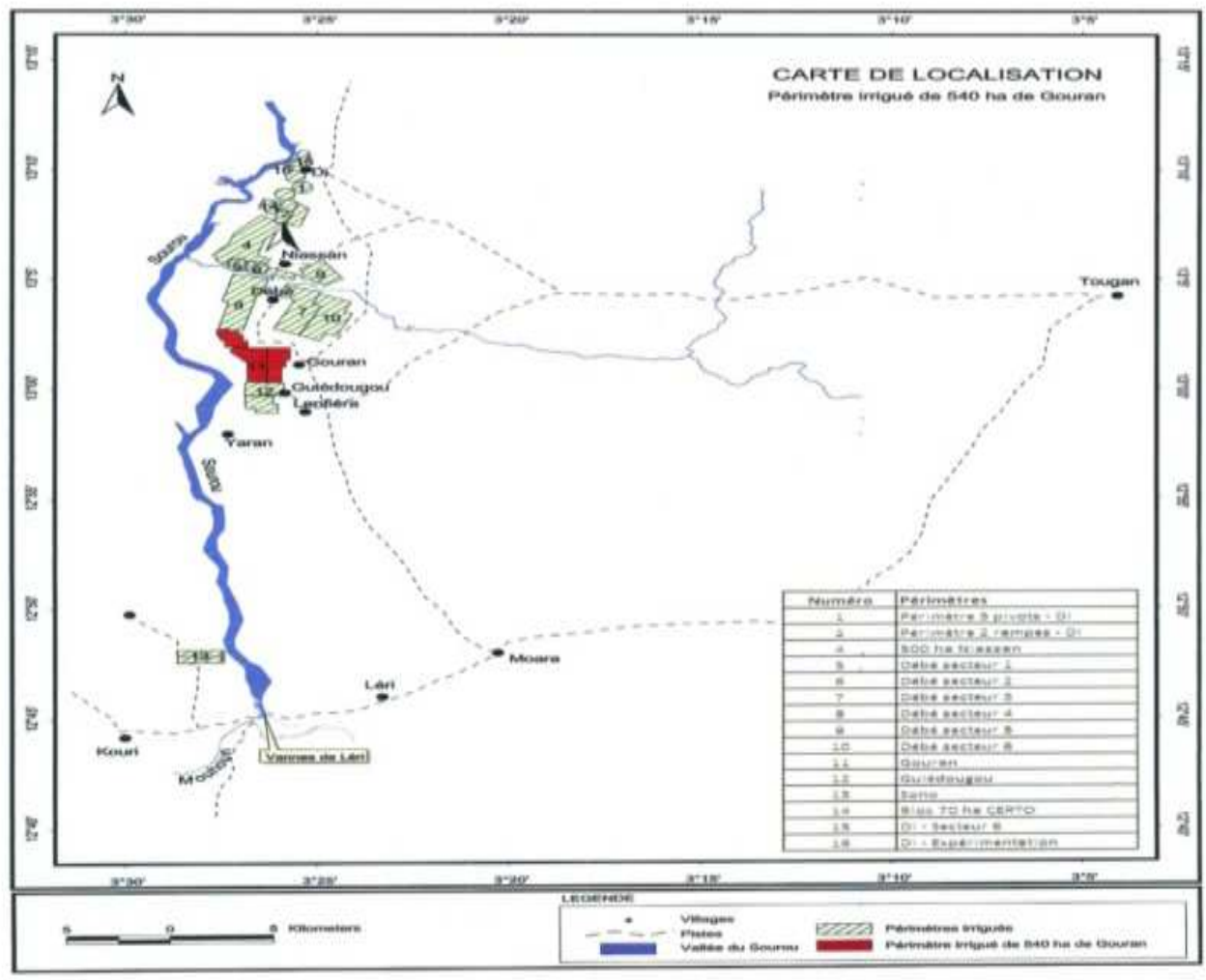

Figure 2 : Localisation du périmètre Irrigué de Gouran. 


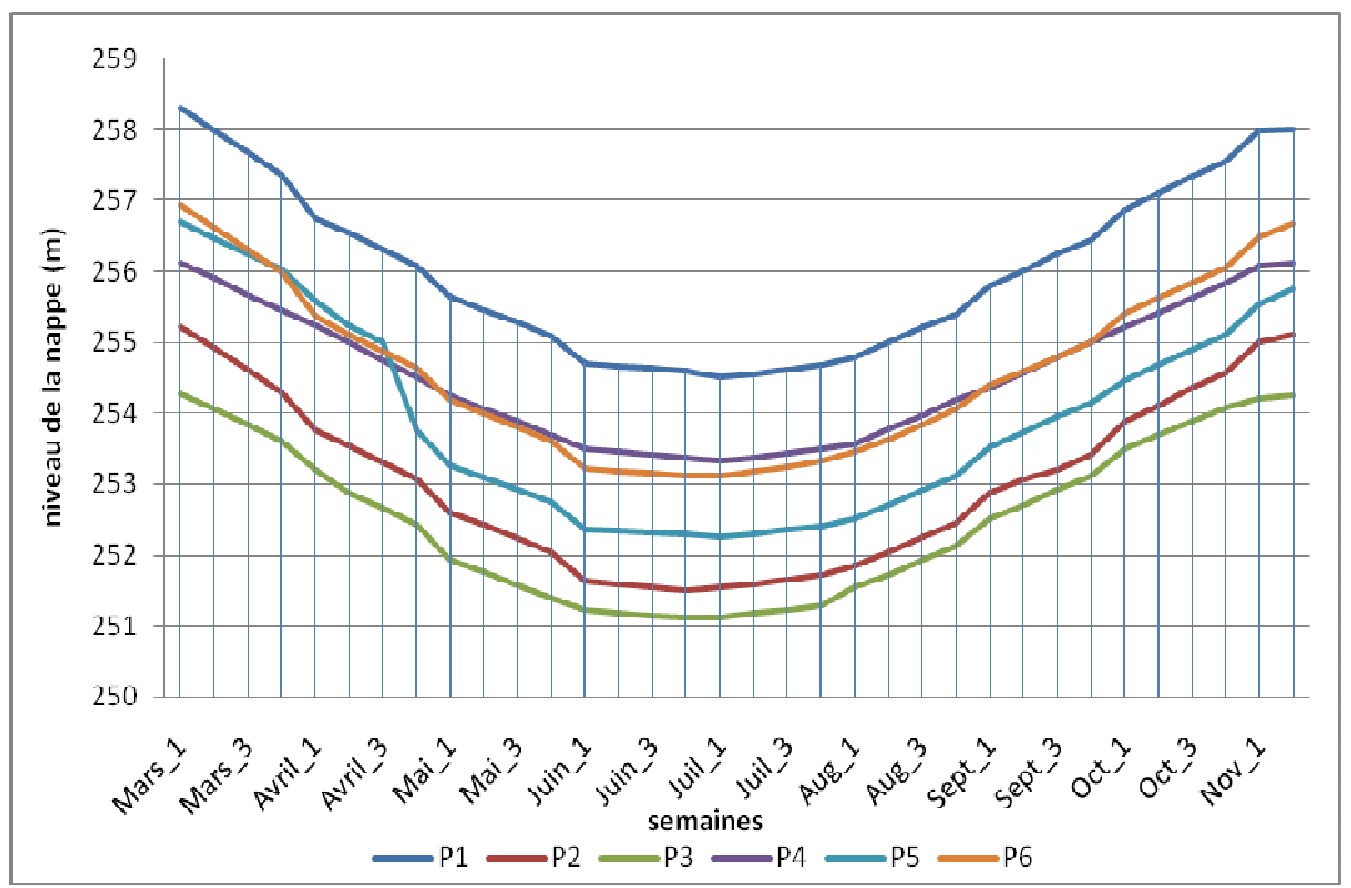

Figure 3 : Fluctuation du niveau de la nappe dans les piézomètres.

\section{Conclusion}

Les contraintes majeures de la productivité des sols des régions tropicales sont en grande partie liées au faible niveau de fertilité de ses sols. La présente étude visait à contribuer à l'accroissement de la production du riz dans le périmètre irrigué de Gouran (situé dans la vallée de Sourou au Burkina Faso), à travers l'adoption d'une meilleure option de fertilisation dans les rizières irriguées combiné à un contrôle de la nappe phréatique sous-jacente. Ceci a permis de déterminer les éléments minéraux limitant la production dans le périmètre dont les types des sols ont été identifiés. Les résultats ont montré que le phosphore et l'azote constituent les éléments limitatifs de la production rizicole. Ces résultats permettent de recommander l'application de la fumure organique bien décomposée (5 t/ha) qui est nécessaires non seulement à l'amélioration de la structure et de la porosité de sols, mais aussi à la maîtrise du $\mathrm{pH}$ pour éviter la tendance à l'acidification. Une formule de fertilisation adaptée au statut chimique des sols déterminé par un suivi régulier de l'évolution de la fertilité des sols sous irrigation est également à recommander.

Ces deux recommandations doivent être associées au contrôle du niveau de la nappe qui permet d'éviter les risques liés à la salinisation.

\section{REFERENCES}

Adam YN. 2000. Etude d'une fumure à base de burkinaphosphate sur le riz irrigué à la Vallée du Kou, dans l'Ouest du Burkina Faso. Mémoire de fin d'études de l'IDR, Université Polytechnique de Bobo Dioulasso, $73 \mathrm{p}$.

Amadji G. 1999. Cours de pédologie, Faculté des Sciences Agronomiques, Université Nationale du Bénin,

AMVS. 2010. Plan stratégique 2010-2025, Di ; AMVS, 49p.

Bandaogo A. 2010. Amélioration de la fertilisation en riziculture irriguée dans la vallée du Kou à travers la technologie du 
placement profond des'granules d'urée, mémoire. DEA Université Polytechnique de Bobo Dioulasso, pp 66

Boko, Kpagbin. 1996. Dégradation des Terres, Restructuration de l'Espace Agraire et Urbanisation au Bas Benin. Margraf, Verlag: Weikersheim ; 190.

BUNSOLS. 1987. Méthodes d'analyse physique et chimique des sols, eaux et plantes, document technique $\mathrm{n}^{\circ} 3$.

BUNASOLS. 1989. Méthodologie de prospection pédologique; BUNASOLS, $115 \mathrm{p}$.

Camille C. 1997. Les Matériels de Fertilisation et de Traitement des Cultures: Technologies de l'Agriculture (vol. 4/5, $1^{\text {ère }}$ édn). Collection FORMAGRI; 343p.

Cassman KG, Peng S, Olk DC, Ladha JK, Reichardt W, Dobermann A, Singh U. 1998. Opportunities for increased nitrogen-use efficiency from improved resource management in irrigated rice systems. Field Crops Research, 56(1-2): 7-39.

CIRAD - GRET. 2006. Mémento de l'Agronome. CIRAD-GRET: Paris ; 799_ 811.

CPCS. 1967. Classification Française des Sols. Inéra Paris Grignon Edition: Paris; 87.

Dobelmann JP. 1976. Technique vivantes. Riziculture pratique 1. Riz irrigué. 220p.

FAO. 1994. Directives pour la description des sols; édition révisée, FAO.

FAO. 2002. Guidelines on Integrated Soil and Nutrient Management and Conservation for Farmer Fields schools. FAO, Land ant plant nutrition Management service and Land and water Developpment Division: Rome, Italie; 164.

Heller. 1969. Quantitative Evaluation of Soil Fertility and the Response to Fertilizers. Wageningen Agricultural, University, Wageningen: The Netherlands; 36.
IFDC. 2008. Fertilizer and new demands: food, feed, fiber - Now fuel. IFDC, 81p.

Kramer PJ. 1969. Plant and Soil Water Relationships: A Modern Synthetics. MC Graw-hill, Book Company: New York; 482.

Lavigne-Delville. 1996. Gérer la fertilité des terres dans les pays du sahel. Diagnostic et conseil aux paysans. Collection «le point sur », 397p.

MCA. 2010. Etudes techniques 'aménagement de 2033ha du périmètre aménageable de Di ; MCA.

Narteh LT, Sahrawat KL. 1997. Potentially mineralizable nitrogen in West African lowland rice soils. Geoderma, 76(1-2): 145-154.

ORSTOM. 1976. Avant-projet détaillé du périmètre de DEBE. ORSTOM : Paris ; $124 \mathrm{p}$

Pieri C. 1989. Fertilité des Terres de Savane. Bilan de Trente ans de Recherches et de Développement au Sud du Sahara. Ministère de la coopération française et CIRAD/ IRAT : Montpellier, France, 444p.

Pèlerin SYSC 2009. La matière organique du sol : un indicateur de la fertilité. Application aux zones sahélienne et soudanienne. Agriculture et Développement, 8: 35-41.

Segda Z. 2006. Gestion de la fertilité du sol pour une production améliorée et durable du riz (Orizasativa L.) au Burkina Faso. Cas de la plaine irrigué de Bagré. Thèse présenté à l'UFR/SVT. Thèse doctorat UO.BF, 202p.

Traoré K, Toé MA. 2008. Capitalisation des initiatives sur les bonnes pratiques agricoles au Burkina Faso, Ouagadougou, $99 \mathrm{p}$.

Vander P. 1994. Farmers motivation for collective action in irrigation : a statistical approach applied to the Office of Niger and Mali. Irrigation and Drainage, 57: 139-150.

Yoshida S. 1981. Fundamentals of rice crop science IRRI. Los Banos, (Phillipines). 113-115p 